\title{
COMPARISON OF MICROWAVE BLACK-BODY TARGET RADIOMETRIC MEASUREMENTS*
}

\author{
D.K. Walker, D. Gu, K. MacReynolds, R. Direen, J. Randa, A.E. Cox, D. Houtz, and R.L. Billinger \\ National Institute of Standards and Technology, Boulder, Colorado 80305, USA
}

\begin{abstract}
1. ABSTRACT
Accurate characterization of the brightness temperature $\left(\mathrm{T}_{\mathrm{B}}\right)$ of black-body targets used for calibrating microwave remote-sensing radiometers includes many inputs: antenna pattern and loss, target temperature, target emissivity, mechanical alignment, and radiometric $T_{B}$ measurements, all of which must be calibrated against physical standards. Here, we describe measurements made using several black-body targets and two different antennas within the WR-42 (18 to $26.5 \mathrm{GHz}$ ) waveguide band. Uncertainty estimates are also shown for the retrieved target $T_{B}$ measurements.
\end{abstract}

Index Terms - Radiometer calibration, measurement uncertainty, calibration target

\section{INTRODUCTION}

Many realizations of microwave brightnesstemperature standards exist in the form of heated or cooled calibration targets, but none is maintained as a national standard by a National Measurement Institute (NMI). This is in contrast to the visible and infrared (IR) portions of the spectrum, in which radiance standards exist and have proven to be very useful [1]

\footnotetext{
*U.S. Government work, not subject to U.S. copyright.
}

A national microwave brightness-temperature standard based on fundamental physical quantities would provide a constant reference for comparison of different instruments over years or decades. Such a stable, accessible reference would benefit studies of long-term phenomena such as climate monitoring, as well as potentially improving numerical weather prediction forecasting.

As part of NIST's ongoing development work on brightness-temperature standards, we have measured combinations of three different black-body targets used for calibration and test purposes by NASA Goddard Space Flight Center. The targets were measured with two different antennas used with our WR-42 thermal noise radiometer over the 18 to 26.5 $\mathrm{GHz}$ frequency range.

\section{METHODOLOGY}

The measurement methodology used is similar to that described in [2]. In summary, it involves measurements performed in an anechoic chamber with floor, ceiling, and walls covered with pyramidal carbon-loaded foam absorber. The chamber's reflection coefficient was measured across the WR-42 
band with a vector network analyzer (VNA) to confirm that the background reflections were below $-30 \mathrm{~dB}$ return loss. Two antennas were characterized; the first was a WR-42 standard-gain horn with half-power beam width of approximately twelve degrees and the second was a conical horn with half-power beam width of approximately fourteen degrees. Each antenna was first measured on a pattern range at 0.5 degree increments in azimuth and elevation. Their ohmic losses were estimated by means of calculations based on the antenna's geometry and the metal resistivity of the antenna bore.

In the work of [2], we found that one potential source of error in our target T_B measurements was the alignment between the antenna axis and the target. Previously, this alignment was performed with a laser beam aimed along the edge of the antenna. The alignment accuracy of this arrangement wasn't established then, but current estimates are that it could have introduced errors on the order of a degree between the antenna axis and the target center. Recently, we have constructed a rigid frame for mounting the antenna in a manner that allows sighting directly through the antenna bore with an optical theodolite. The target being measured is mounted on a six-axis micropositioner. The target is fitted with a temporary cross-hair "bull's eye" to allow antennatarget alignment. A post-check of the alignment over the entire longitudinal range of the positioner (about $4.5 \mathrm{~m}$ ) confirmed that the alignment accuracy is now better than 2 arc-sec, or approximately $2 \mathrm{~mm}$ lateral misalignment at $4.5 \mathrm{~m}$ range.
Another potential source of error in our previous work was that $\eta$, the fraction of the antenna pattern subtended by the target, was calculated from the farfield antenna pattern with no correction made for nearfield effects [3]. In the present work, we compared our results with and without a near-field correction.

Three targets were measured with each antenna. The targets each consist of an array of tetrahedral pyramids of iron-loaded epoxy mounted on a circular aluminum substrate with backside heating element and embedded thermometers for temperature control and target monitoring. Two targets, designated MIR-1 and MIR2 , were $18 \mathrm{~cm}$ in diameter. These two targets differ in that MIR-1 has pyramids of $3 \mathrm{~cm}$ height on a $1 \mathrm{~cm}$ grid with flat valleys between the bases from the machining process, while MIR-2 has pyramids of $3 \mathrm{~cm}$ height on a $0.7 \mathrm{~cm}$ grid with sharp valleys at the bases. The third target, designated MIR-3, was $33 \mathrm{~cm}$ in diameter with pyramids of $4 \mathrm{~cm}$ height on a $1 \mathrm{~cm}$ grid, also with sharp valleys at the bases.

We also monitored the background (chamber) temperature during operation. Depending on the target being tested, foam absorber was used to surround the circular target face to reduce unwanted reflections from the backing plate or the positioner. Each target was measured at multiple ranges, from approximately $0.5 \mathrm{~m}$ up to $4.5 \mathrm{~m}$ between antenna and target, while heated to around $340 \mathrm{~K}$ as well as at ambient temperature as a check.

The NIST WR-42 noise-temperature radiometer [2] was used for all T_B measurements. After calibrating 
the radiometer system, we measured the reflection coefficient and microwave noise temperature of the device (comprising the waveguide feed, antenna, and chamber with target) forward from the measurement reference plane. The target brightness temperature and associated uncertainties were then calculated.

\section{RESULTS}

A representative radiometric measurement of one of the three black-body targets, with a pyramidal standard-gain horn attached to the WR-42 waveguide radiometer is shown in Figure 1, with $\eta$ calculated by means of the far-field antenna pattern for all distances. The measured brightness temperature was then compared to the brightness temperature computed from the target physical temperature and approximate emissivity of the target. The calculated target temperature varies beyond the limits of our uncertainties (error bars) at some closer distances. Figure 2 shows the same target-antenna measurements except that a near-field antenna pattern correction is now used to correct $\eta$ at each distance. The calculated target temperature now falls within the limits of our uncertainties for the improved antenna pattern calculation.

The near-field antenna pattern correction can be a significant part of the error in $\eta$. The following two figures show the calculated near- and far-field $\eta$ for the same pyramidal horn. Figure 3 is for the MIR-1 and MIR-2 targets, while Figure 4 is for the MIR-3 target. While not shown here, we found that the shape of the curves for the two target types is only weakly dependent on frequency. However, the general form is strikingly different for the two target sizes due to the rapid spatially-varying modal field amplitudes at close range. The maximum difference seen in the near- vs. far-field $\eta$ for the MIR-1 target (and MIR-2, which is the same diameter) is about $24 \%$ at a range of $0.75 \mathrm{~m}$, while the MIR-3 target shows a maximum difference of about $8 \%$ at a range of $1.5 \mathrm{~m}$.

The uncertainties associated with the measured and predicted target brightness temperatures are observed to depend on the target size and range, along with the $\eta$ calculation (near-field antenna pattern correction applied or not). In our study, we saw that a larger target is preferable not only because of the lower uncertainty due to more signal (i.e. higher $\eta$ ) at a given range, but also because the near- vs. far-field $\eta$ estimates are closer. Our results indicate that this is likely to be antenna-dependent.

\section{ACKNOWLEDGMENT}

The authors gratefully acknowledge the assistance of Paul Racette of NASA GSFC for providing the calibration targets used in this study.

\section{REFERENCES}

[1] J. P. Rice and B. C. Johnson, "NIST activities in support of space-based radiometric remote sensing," Proc. SPIE, vol 4450, pp. $108-126,2001$.

[2] J. Randa, A.E. Cox, and D.K. Walker, "Proposal for Development of a National Microwave Brightness-Temperature Standard," Proc. SPIE, Vol. 6301, 630105 (Sept. 2006).

[3] R. C. Wittman, A. E. Cox, and R. H. Direen, "Proximity Effects in the Calibration of Microwave Radiometers," Proc. Antenna Meas. Tech. Soc., pp. 333-336, Oct. 2003. 


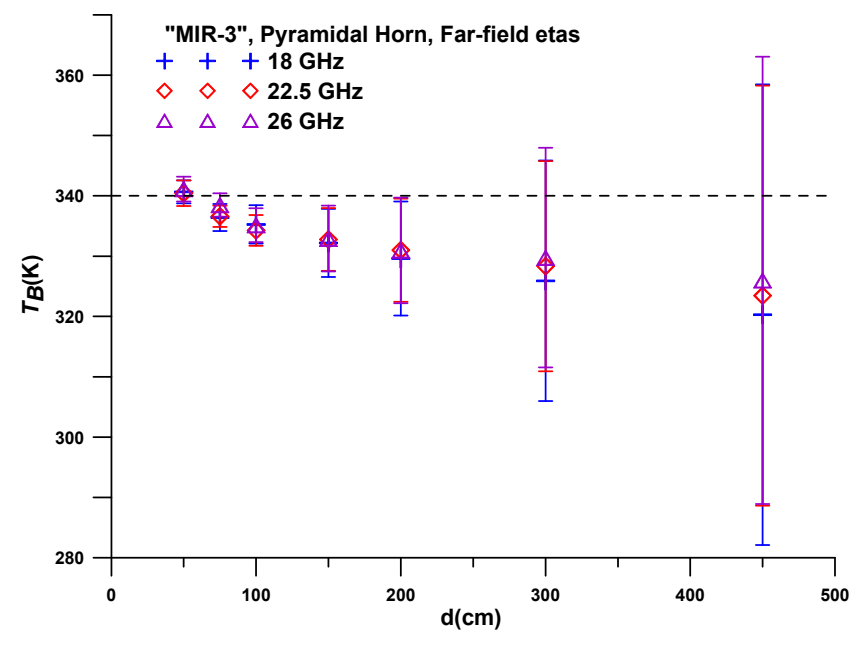

Figure 1. Calculated MIR-3 radiometric target temperature vs. distance in which the far-field antenna pattern for $\eta$ (eta) was used. The approximate surface temperature of the target is shown by the dashed line.

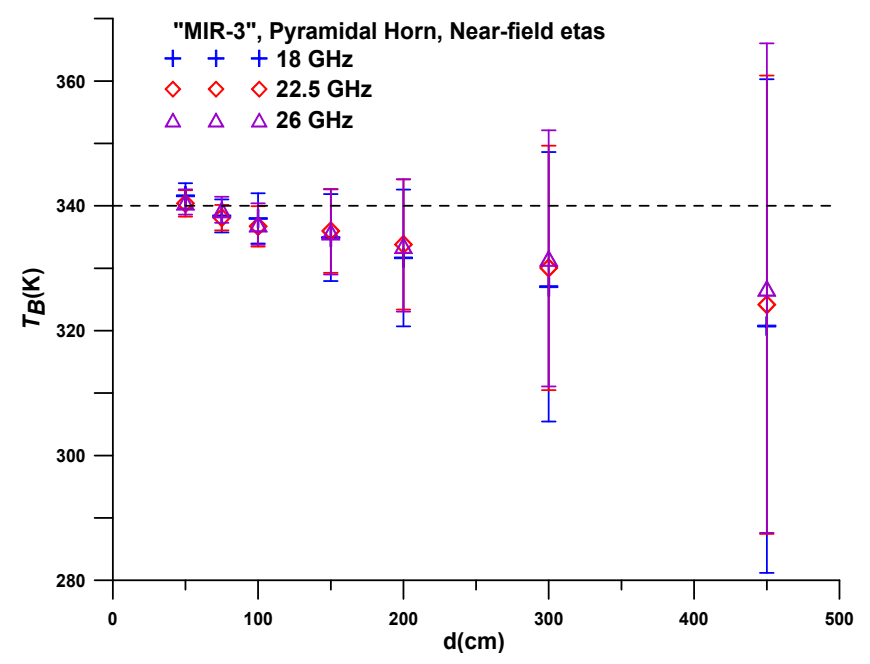

Figure 2. Calculated MIR-3 radiometric target temperature vs. distance with the near-field antenna pattern correction for $\eta$. The approximate surface temperature of the target is shown by the dashed line.

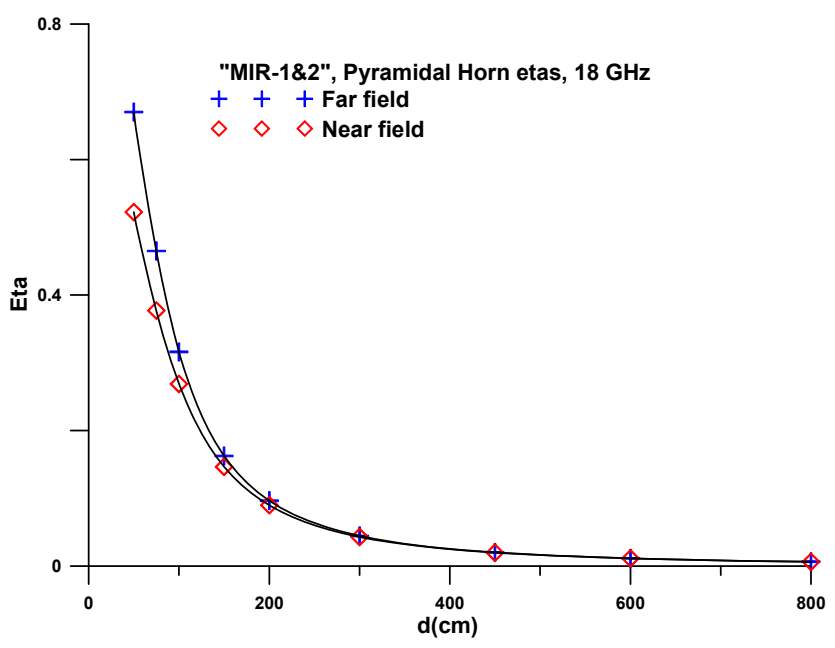

Figure 3. Comparison of $\eta$ vs. target distance for near- and far-field antenna patterns with MIR-1 \& MIR-2 targets at $18 \mathrm{GHz}$.

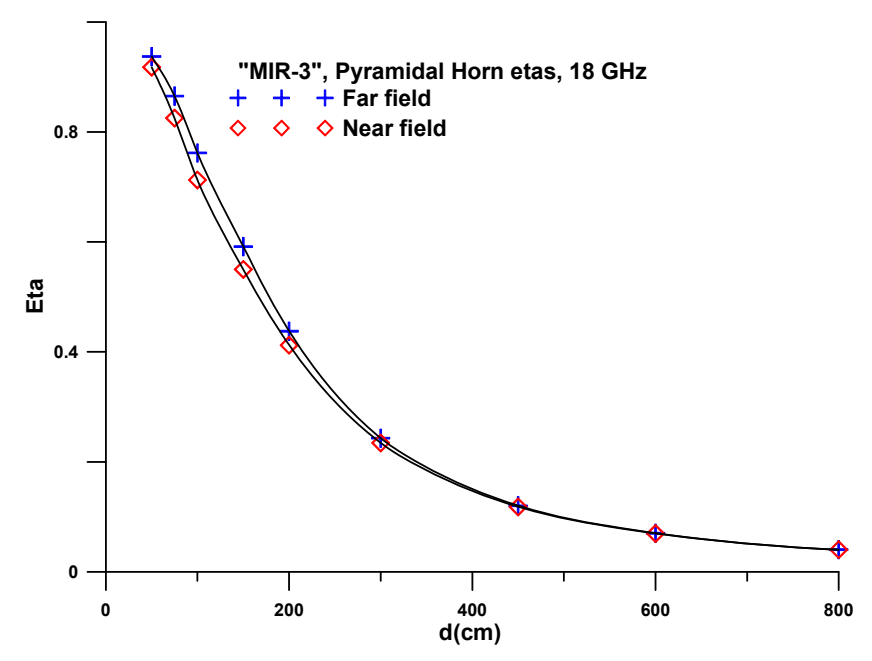

Figure 4. Comparison of $\eta$ vs. target distance for near- and far-field antenna patterns with MIR-3 target at $18 \mathrm{GHz}$. 